FACTA UNIVERSITATIS (NIŠ)

Ser. Math. Inform. Vol. 36, No 3 (2021), 461 - 474

https://doi.org/10.22190/FUMI190318034A

Original Scientific Paper

\title{
NEW RESULTS ON SEMICLOSED LINEAR RELATIONS
}

\author{
Gherbi Abdellah ${ }^{1}$, Messirdi Bekkai ${ }^{1}$ and Messirdi Sanaa ${ }^{2}$ \\ 1 Oran's Hight School of Electrical and Energetic Engineering, \\ Laboratory of Fundamental and Applicable Mathematics of Oran (LMFAO), \\ University of Oran 1, 31000 Oran, Algeria \\ 2 High Institute ISIPS, Hainault, Belgium
}

\begin{abstract}
This paper has triple main objectives. The first objective is an analysis of some auxiliary results on closedness and boundedness of linear relations. The second objective is to provide some new characterization results on semiclosed linear relations. Here it is shown that the class of semiclosed linear relations is invariant under finite and countable sums, products, and limits. We have obtained fundamental new results as well as a Kato Rellich Theorem for semiclosed linear relations and essentially interesting generalizations. The last objective deals with semiclosed linear relation with closed range, where we have particularly established new characterizations of closable linear relation.
\end{abstract}

Keywords: Semiclosed linear relation, Closable, Countable sums and products, Limits, Kato Rellich Theorem, Closed range.

\section{Introduction}

Let $H$ be a complex Hilbert space with its scalar product and associated hilbertian norm denoted by $\langle. ;$.$\rangle and \|$.$\| , respectively. A linear relation or multivalued$ linear operator $T$ is a linear mapping with linear domain $\mathcal{D}(T) \subseteq H$, that assigns to each $x \in \mathcal{D}(T)$ a nonempty set $T x=\{y:(x, y) \in G(T)\} \subset H$. If $T x$ never contains more then one element, then $T$ is (single-valued) linear operator

Received March 18, 2019. accepted May 03, 2021.

Communicated by Dragana Cvetković-Ilić

Corresponding Author: Gherbi Abdella, Oran's High School of Electrical and Energetic Engineering, Laboratory of Fundamental and Applicable Mathematics of Oran (LMFAO), University of Oran 1, 31000 Oran, Algeria | E-mail: gherbi1@live.fr 2010 Mathematics Subject Classification. 47A06

(C) 2021 by University of Niš, Serbia | Creative Commons License: CC BY-NC-ND 
on $H$. Note that $G(T)$ is the graph of $T$ and it is a subset of $H \times H$ defined by $G(T)=\{(x, y) \in H \times H: x \in \mathcal{D}(T), y \in T x\}$. The range $\mathcal{R}(T)$ of $T$ is defined as the union of all $T x, x \in \mathcal{D}(T)$. The null space $\mathcal{N}(T)$ and the multivalued part $T(0)$ of the linear relation $T$ are respectively defined by

$$
\mathcal{N}(T)=\{x \in H:(x, 0) \in G(T)\} \text { and } T(0)=\{y \in H:(0, y) \in G(T)\} .
$$

If $\mathcal{N}(T)=\{0\}$ (resp. $\mathcal{R}(T)=H$ ), we say that $T$ is injective (resp. surjective). If $T$ is injective and surjective, we say that it is a bijection. Let $L R(H)$ denotes the space of all linear relations on $H$.

Proposition 1.1. [3],[11] Let $T \in L R(H)$. Then:

$$
\begin{gathered}
\mathcal{N}(T) \times\{0\}=G(T) \cap(H \times\{0\}) ; \\
\{0\} \times T(0)=G(T) \cap(\{0\} \times H) ; \\
H \times \mathcal{R}(T)=G(T)+(H \times\{0\}) ; \\
\mathcal{D}(T) \times H=G(T)+(\{0\} \times H) .
\end{gathered}
$$

For every $T \in L R(H)$, there exists a relation $T^{-1} \in L R(H)$ called the formal inverse of $T$ defined by $G\left(T^{-1}\right)=\{(y, x):(x, y) \in G(T)\}$. Obviously,

$$
\mathcal{D}\left(T^{-1}\right)=\mathcal{R}(T), \mathcal{R}\left(T^{-1}\right)=\mathcal{D}(T), \mathcal{N}\left(T^{-1}\right)=T(0) \text { and } T^{-1}(0)=\mathcal{N}(T) .
$$

The adjoint $T^{*}$ of $T$ is defined by

$$
G\left(T^{*}\right)=\{(y, x):\langle v, y\rangle=\langle u, x\rangle \text { for some }(u, v) \in G(T)\} .
$$

If $S$ and $T$ are two relations in $L R(H)$, then the sum $S+T$ and the product $S T$ are also relations in $L R(H)$ and they are respectively defined by:

$$
\begin{aligned}
G(S+T) & =\{(x, u+v):(x, u) \in G(S) \text { and }(x, v) \in G(T)\} \\
G(S T) & =\{(x, y):(x, v) \in G(T) \text { and }(v, y) \in G(S) \text { for some } v \in H\} .
\end{aligned}
$$

The identity relation defined on a nonempty subset $M$ of $H$ will be denoted by $I_{M}$.

For all $T \in L R(H)$, let $Q_{T}$ denote the natural quotient map from $H$ onto $H / \overline{T(0)}$ where $\overline{T(0)}$ is the closure of $T(0)$. Note that the quotient map $Q_{T}$ is used to extend the definition of the operator norm to the linear relations class. Clearly $T_{s}=Q_{T} T$ is a linear operator with $\mathcal{D}\left(T_{s}\right)=\mathcal{D}(T)$. $T_{s}$ is called a linear operator part (or a single valued part) of $T$. For $x \in \mathcal{D}(T),\|T x\|=\left\|T_{s} x\right\|$ and the norm of $T$ is defined by $\|T\|=\left\|T_{s}\right\|$. A relation $T$ is said to be continuous if $\|T\|<\infty$. If $T$ is continuous with $\mathcal{D}(T)=H$, then we say that $T$ is bounded. Given two relations $S, T \in L R(H)$, we say that $T$ is an extension of $S$ if

$$
T_{\mid \mathcal{D}(S)}=S .
$$

Clearly, if $T$ is an extension of $S$, then $G(S) \subset G(T)$. However, the converse is not true in general only if $T(0)=S(0)$. 
One main reason why linear relations are more convenient than operators is that one can define the inverse, the closure, the conjugates and the completion for a linear relation without any additional condition on the relation. See for example [3] and [1] for interesting works on linear relations.

We investigate in this paper the notion of semiclosed linear relations on Hilbert and Banach spaces, also called paracomplete linear relations by Alvarez and Wilcox in [2]. Paracomplete subspaces in Banach spaces were studied in the papers [4], [5], [10] and others. The notion of a semiclosed, or almost closed or quotient, operator introduced in [6], [7], [8] and [12] can be naturally generalized to linear relations. The class of semiclosed linear relations is closed under addition, product, inversion, restriction, and limits. We give some interesting new characterizations of these relations and we obtain certain interesting generalizations of results on the closedness, boundedness, product and some of semiclosed linear relations. Finally we establish a certain number of results concerning the closedness of $\mathcal{R}(T)$ where $T$ is a semiclosed linear relation by using Neubauer's Lemma. The structure of this work is as follows. Throughout Section 2, we give some auxiliary results on linear relations, sometimes purely algebraic and topological, which are required in the sequel. In section 3, we define and obtain several properties of semiclosed linear relations via the concept of selection or single valued part of a linear relation in Hilbert spaces. A linear relation with semiclosed multivalued part is semiclosed if and only if it has a semiclosed selection. We considered the case where a semiclosed linear relation is closed, closable or bounded. Restriction, inverse, adjoint, finite sum, product and iteration of semiclosed linear relations are also studied as well as a Kato Rellich Theorem for semiclosed linear relations. Finally, in Section 4, we investigate semiclosed linear relations with closed range which gives in particular a new characterization of closable linear relations.

\section{Some auxiliary results on linear relations}

We commence with a recollection of some preliminary properties required in the sequel.

A relation $T \in L R(H)$ is said to be closed if its graph is closed in $H \times H$. The closure of $T$ is the relation $\bar{T} \in L R(H)$ defined by $G(\bar{T})=\overline{G(T)}$. Hence, $T$ is closed if $T=\bar{T}$.

Lemma 2.1. [3] Let $T \in L R(H)$. Then, $T$ is closed if and only if $T_{s}$ is closed linear operator and $T(0)$ is a closed subspace of $H$.

Let $H_{T}$ denote the vector space $\mathcal{D}(T)$ endowed with the graph inner product $\langle., .\rangle_{T}$ of $T$ defined by

$$
\langle x, y\rangle_{T}=\langle x, y\rangle_{H}+\langle T x, T y\rangle_{H} \text { for } x, y \in \mathcal{D}(T) .
$$

Clearly, $H_{T}=H_{T_{s}}$, also $H_{T}$ is norm isomorphic to $G(T)$ when $T$ is a linear operator. Thus, we have: 
Proposition 2.1. Let $T$ be a densely defined linear relation on $H$ with $T(0)$ is closed, then $T$ is closed if and only if $H_{T}$ is complete.

Proof. One only has to see that $H_{T}=H_{T_{s}}$ which is norm isomorphic to the closed graph $G\left(T_{s}\right)$ in $H \times H / T(0)$.

Proposition 2.2. If $T$ is a closed relation. Then $T$ is assimilable to a continuous relation from $H_{T}$ into $H$.

Indeed, let $i: H_{T} \hookrightarrow H$ be a linear operator defined by:

$$
\mathcal{D}(i)=H_{T} \text { and } i(x)=x \text { for all } x \in H_{T} .
$$

( $i$ is an injection mapping from $H_{T}$ onto $H$ ). Now we need to show that the relation $T i$ is of a finite norm:

$$
\|T i\|=\sup _{x \in H_{T}} \frac{\|(T i) x\|}{\|x\|_{T}}=\sup _{x \in \mathcal{D}(T)} \frac{\|T x\|}{\|x\|+\|T x\|}= \begin{cases}\frac{\|T\|}{1+\|T\|} & \text { if }\|T\|<+\infty \\ 1 & \text { if }\|T\|=+\infty\end{cases}
$$

Corollary 2.1. If $T$ is continuous such that $\mathcal{D}(T)$ and $T(0)$ are closed, then $T$ is closed.

A linear relation $T$ is said to be closable if $\bar{T}$ is an extension of $T$.

Lemma 2.2. [3] Let $T \in L R(H)$. The following properties are equivalent:

1. $T$ is closable;

2. $T(0)=\bar{T}(0)$;

3. $T_{s}$ is closable and $T(0)$ is closed.

Proposition 2.3. If $T$ is closable linear relation, then $\mathcal{D}(\bar{T})=\overline{\mathcal{D}(T)}$ and $T$ is continuous on $\mathcal{D}(\bar{T})$.

Proof.

$$
\overline{\mathcal{D}(T)}=\overline{\mathcal{D}\left(T_{s}\right)}=\mathcal{D}\left(\overline{T_{s}}\right)=\mathcal{D}\left((\bar{T})_{s}\right)=\mathcal{D}(\bar{T}) .
$$

Hence $\mathcal{D}(\bar{T})$ is closed and using the closed graph theorem for linear relations ([3] Theorem III.4.2) we obtain that $\bar{T}$ is continuous.

\section{Main results on semiclosed linear relations}

\subsection{Characterization of semiclosed linear relation}

A linear subspace $M$ of a Hilbert space $H$ is called semiclosed if there exists a norm $\|\cdot\|_{M}$ such that $\left(M,\|\cdot\|_{M}\right)$ is complete and continuously embedded in $H$, i.e, $\|x\| \leq \lambda\|x\|_{M}$ for any $x \in M$.

In the two following theorems, we collect some well known characterizations and properties of semiclosed linear subspaces in a Hilbert space $H$. 
Theorem 3.1. [9] Let $M$ be a linear subspace of $H$. The following statements are equivalent:

1. $M$ is semiclosed subspace of $H$.

2. $M$ is the range of a bounded operator on $H$.

3. $M$ is the range of a closed operator on $H$.

4. $M$ is the domain of a closed operator on $H$.

Theorem 3.2. [11] Let $M, N$ be two linear subspaces of $H$. Then:

1. $M$ and $N$ are semiclosed subspaces of $H$ if and only if $M \times N$ is a semiclosed subspace of $H \times H$.

2. If $M$ and $N$ are semiclosed subspaces of $H$, then $M+N$ and $M \cap N$ are also semiclosed subspaces of $H$.

3. Neubauer's Lemma: If $M, N$ are semiclosed subspaces and both of $M+N$ and $M \cap N$ are closed, then $M$ and $N$ are closed in $H$.

A semiclosed linear relation can also be characterized by means of semiclosed subspaces.

Definition 3.1. A linear relation $T \in L R(H)$ is said to be semiclosed on $H$ if its graph $G(T)$ is semiclosed in $H \times H$.

Let $S C(H)$ denote the set of all semiclosed linear relations on $H$.

Corollary 3.1. Let $T \in S C(H)$. Then, $\mathcal{D}(T), N(T), \mathcal{R}(T)$ and $T(0)$ are semiclosed sets in $H$.

Proof. The proof follows immediately from the proposition 1.1 and the theorem 3.2 .

A linear operator $A$ is called a selection (or single valued part) of $T$ if

$$
T=A+T-T \text { and } \mathcal{D}(A)=\mathcal{D}(T) .
$$

In particular, a linear operator is a selection of itself. The singlevalued part $T_{s}$ of a linear relation $T$ is a natural selection of $T$, nevertheless, $T$ admits other selections.

Proposition 3.1. [3] Let $A$ be a selection of T. Then

1. $\mathcal{R}(T)=\mathcal{R}(A)+T(0)$. However, this sum may not always be direct.

2. $G(A) \cap(\{0\} \times T(0))=\{0\} \times\{0\}$. 
3. $G(T)=G(A)+(\{0\} \times T(0))$.

One of the basic results of this paper is the following:

Theorem 3.3. Let $T$ be a linear relation with $T(0)$ semiclosed in $H$. Then, $T$ is semiclosed linear relation if and only if $T$ has a semiclosed selection.

Proof. Let $T$ be a semiclosed linear relation, then $T(0)$ is semiclosed in $H$. Let $P$ be the linear projection defined on $\mathcal{R}(T)$ such that $\mathcal{N}(P)=T(0)$. Then we have in one hand,

$$
P T(0)=\{0\} \text {, i.e } P T \text { is a linear operator satisfying } \mathcal{R}(P T) \cap T(0)=\{0\} .
$$

In the other hand, we have for all $y \in T x$ :

$$
T x=y+T(0)=P y+(I-P) y+T(0)=P T x+T(0) \text {. }
$$

Hence, $T=P T+T-T$ and $G(T)=G(P T)+(\{0\} \times T(0))$. Thus $T=P T \oplus T(0)$, therefore $P T$ is a semiclosed selection of $T$.

Conversely, let $A$ be a semiclosed selection of $T$. Then $T=A+T-T$, where $T-T$ is a linear relation defined by:

$$
G(T-T)=\{0\} \times T(0) .
$$

Since $G(T)=G(A)+(\{0\} \times T(0))$ we obtain, $G(T)$ is semiclosed in $H \times H$, hence $T$ is semiclosed linear relation.

The Proposition 1.8 of [2] is now an immediate consequence of the Theorem 3.3, where the authors supposed that $T(0)$ is closed. Indeed, it is shown in [2] that if $T \in L R(H)$ with $T(0)$ closed, then $T$ is semiclosed if and only if $T_{s}=Q_{T} T$ is semiclosed. The theorem 3.3 generalizes this situation where $T(0)$ is considered only semiclosed.

So, since $H_{T_{s}}=H_{T}$, combining the definition 2 in [12] and Proposition 1.8 of [2], we deduce the following characterization result which is in fact, a natural generalization of Theorem 4.2 of [13].

Proposition 3.2. Let $T \in L R(H)$ with $T(0)$ closed. Then $T$ is semiclosed if and only if there exists a inner product $(.,$.$) such that H_{T}=(\mathcal{D}(T),(.,)$.$) is complete,$ $H_{T} \hookrightarrow H$ and $T$ is continuous from $H_{T}$ to $H . H_{T}$ is called the auxiliary Hilbert space of $T$.

Similarly, if $T$ is a linear relation on a Banach space $E$ with closed multivalued part, then we say that $T$ is semiclosed on $E$ if and only if there exists a norm $\|\cdot\|_{T}$ on $\mathcal{D}(T)$ such that $E_{T}=\left(\mathcal{D}(T),\|\cdot\|_{T}\right)$ is a Banach space continuously embedded in $E$ and $T$ is continuous from $E_{T}$ to $E$.

Some essential characterizations on semiclosedness of linear relations are given below. 
Proposition 3.3. Let $T \in S C(H)$ such that both of $\mathcal{D}(T)$ and $T(0)$ are closed, then $T$ is bounded.

Proof. We have from the theorem 3.3, that $T_{s}$ is semiclosed linear operator with $\mathcal{D}\left(T_{s}\right)=\mathcal{D}(T)$. Thus, there exists an inner product (.,.) on $\mathcal{D}(T)$ such that the Hilbert space $H_{T_{s}}=H_{T}=(\mathcal{D}(T),(.,)$.$) is continuously embedded in H$ and $T_{s}$ is bounded from $H_{T_{s}}$ to $H$. Since $\mathcal{D}(T)=\mathcal{D}\left(T_{s}\right)$ is closed, we obtain $\mathcal{D}(T)=H$ and $T_{s}$ is bounded on $H$. Hence, $T$ is bounded linear relation with $T(0)$ closed. Consequently, $T$ is bounded closed linear relation.

Obviously, every closed linear relation is semiclosed. Nevertheless, there exists semiclosed linear relations which are not closed. Indeed, the fact that $T$ is semiclosed linear relation prove that $T(0)$ is a semiclosed subset in $H$, however $T(0)$ is not necessarily closed. Consequently, $T$ is not necessarily closed.

The following proposition gives an important case of semiclosed linear relations which are not closed on $H$, especially when $\mathcal{D}(T)$ and $\mathcal{R}(T)$ are semiclosed subspaces but non closed.

Proposition 3.4. Let $T \in S C(H)$, then $T^{-1} T$ and $T T^{-1}$ are also semiclosed relations on $H$.

Proof. The result follows immediately from the facts, $T T^{-1}=I_{\mathcal{R}(T)}+T(0)$ and $T^{-1} T=I_{\mathcal{D}(T)}+T^{-1}(0)$.

It may be very important to note that there exists some closable linear relations which are not semiclosed and there exists some semiclosed linear relations which are not closable. Hence, one can confirm that there is no relation in terms of inclusion between the set of semiclosed linear relations and the set of closable linear relations. To clarify this situation, let us consider the two following original examples:

Example 3.1. The space $E=C([a, b])$ of continuous complex valued functions on $[a, b]$, equipped with the norm $\|x\|_{\infty}=\sup _{t \in[a, b]}|x(t)|, x \in E$, is a Banach space. Consider:

$$
T x=\int x(t) d t, x(t) \in E
$$

with the polynomials $\mathcal{P}$ as its domain. $T$ is a linear relation on $E$,

$$
T(0)=\{y \in E:(0, y) \in G(T)\}=\mathbb{C}
$$

where $G(T)=\{(x, y) \in E \times E: x \in D(T)=\mathcal{P}, y \in T x\}$ is the graph of $T$. In particular, $T(0)$ is closed in $E$ since on the complex constant polynomials the norm $\|\cdot\|_{\infty}$ and the absolute value are equivalent. Furthermore,

$$
T=T_{s}+T(0)
$$

where the operator linear part $T_{s}$ of $T$ is given by:

$$
T_{s} x(t)=\int_{a}^{t} x(t) d t
$$


with domain $D\left(T_{s}\right)=D(T)=\mathcal{P}, T_{s} x$ is the primitive function of $x$ which vanishes at the point $t=a$.

$T$ is a closable linear relation on $E$ since $T(0)$ is closed in $E$ and $T_{s}$ is closable on $E$. Indeed, let $\left(x_{n}\right)_{n \in \mathbb{N}}$ be a sequence in $D(T)$ such that $\left(x_{n}\right)_{n \in \mathbb{N}}$ and $\left(T x_{n}\right)_{n \in \mathbb{N}}$ are uniformly convergent to 0 and $y$ respectively, then necessarily $y=0$.

In fact, $T$ is closable but not semiclosed linear relation on $E$, since $T_{s}$ is a non-semiclosed linear operator on $E$.

Assume that $T_{s}$ is semiclosed, then there exists a Banach space $E_{s}$ such that the graph

$$
G\left(T_{s}\right)=\left\{\left(x, T_{s} x\right): x \in \mathcal{P}, T_{s} x \in \mathcal{P}_{0}\right\}, \mathcal{P}_{0}=\{y \in \mathcal{P}: y(a)=0\}
$$

of $T_{s}$ is closed in $E_{s} \times E$. Thus, $G\left(T_{s}\right)$ is a complete metric space. However, $G\left(T_{s}\right)$ is also the union of countably many finite-dimensional subspaces and is thus of first category. By Baire's theorem, complete metric spaces are of second category, which is a contradiction. Thus, the operator $T_{s}$ with domain $\mathcal{P}$ is not semiclosed.

Example 3.2. Consider over the space $C([0,1])$ of all continuous functions on $[0,1]$ equipped with its usual norm, the linear operators $T$ and $S$ defined by: $T=\frac{d}{d x}$ with domain $\mathcal{D}(T)=C^{1}([0,1])$ and $S f(x)=f(0) g(x)$ domain $\mathcal{D}(S)=C([0,1])$ where $g \neq 0$ is arbitrarily fixed in $C([0,1])$. Since $T$ is closed and $S$ is bounded, the product $S T$ defined by $S T f=\frac{d f}{d x}(0) g(x)$ with domain $\mathcal{D}(S T)=\mathcal{D}(T)$ is a semiclosed linear operator on $C([0,1])$. Indeed, it is shown in [12] that the sum and the product of two semiclosed linear operators is also semiclosed. Now let $f_{n}(x)=-\frac{e^{-n}}{n}$. Then, for all $n \in \mathbb{N}^{*}, f_{n} \in \mathcal{D}(S T)$, for all $x \in[0,1],\left|f_{n}(x)\right|^{2}=\frac{e^{-2 n}}{n^{2}} \rightarrow 0$ and $\left|f_{n}(x)\right|^{2} \leq 1$ with $1 \in L^{1}([0,1])$. Using the Lebesgue's dominated convergence theorem, we obtain

$$
\lim _{n \rightarrow+\infty} \int_{0}^{1}\left|f_{n}(x)\right|^{2} d x=\int_{0}^{1} \lim _{n \rightarrow+\infty}\left|f_{n}(x)\right|^{2} d x=0 .
$$

Hence, $\left(f_{n}\right)_{n}$ converge to 0 in $C([0,1])$. In other hand we have $S T f_{n}=\frac{d f_{n}}{d x}(0) g=g \neq 0$. Or, $(0, g)$ can not be in the graph of any linear operator, so $S T$ is not closable.

\subsection{Restriction, inverse and adjoint of semiclosed linear relations}

Theorem 3.4. Let $T \in S C(H)$. Then for all semiclosed subspace $M$ of $\mathcal{D}(T)$, the restriction $T_{\mid M}$ of $T$ to $M$ is a semiclosed linear relation on $H$.

Proof. Let $T \in S C(H)$, then $T(0)$ is semiclosed set in $H$ and there exists a semiclosed selection $A$ of $T$ such that $T=A+T-T$.

Then we have: $T_{\mid M}=T I_{M}, T_{\mid M}(0)=T(0)$ and for all $x \in M, T_{\mid M} x=A_{\mid M} x+T(0)$ where $A_{\mid M}$ is the restriction of $A$ to $M$. Hence, $G\left(T_{\mid M}\right)=G\left(A_{\mid M}\right)+(\{0\} \times T(0))$ is semiclosed subspace of $H \times H$ because $A_{\mid M}$ is semiclosed linear operator on $H$. This complete the proof.

Proposition 3.5. $T \in S C(H) \Leftrightarrow T^{-1} \in S C(H)$. 
Proof. Assume that $T \in S C(H)$ and let $J=\left(\begin{array}{cc}0 & 1 \\ 1 & 0\end{array}\right)$ be a linear operator defined on $H \times H$. Then $J$ is a semiclosed operator on $H \times H$. Clearly, $J(G(T))=G\left(T^{-1}\right)$. Since $T$ is supposed semiclosed, we obtain $J_{\mid G(T)}$ is semiclosed operator. Hence, $\mathcal{R}\left(J_{\mid G(T)}\right)=G\left(T^{-1}\right)$ is a semiclosed subspace of $H \times H$.

Corollary 3.2. Let $T \in S C(H)$. The range and inverse range of any semiclosed subspace of $H$ by $T$ is semiclosed in $H$.

Proposition 3.6. Let $T \in S C(H)$, then $T^{*} \in S C(H)$.

Proof. It follows immediately from the fact that $G\left(T^{*}\right)=[\mathcal{J}(G(T))]^{\perp}$ where $\mathcal{J}=$ $\left(\begin{array}{cc}0 & -i \\ i & 0\end{array}\right)$.

\subsection{Finite sum, product and iteration of semiclosed linear relations}

Theorem 3.5. Let $S, T$ be two semiclosed linear relations and $\alpha \in \mathbb{C}^{*}$. Then: $S+T, S T$ and $\alpha T$ are semiclosed linear relations on $H$.

Proof. Let $A$ and $B$ be two semiclosed selections of $S$ and $T$ respectively. Since $(S+T)(0)=S(0)+T(0)$ is semiclosed subset of $H$, it will be sufficient to show that $S+T$ has a semiclosed selection in order to prove that $S+T$ is semiclosed linear relation. Recall that the domain $\mathcal{D}_{+}$of $S+T$ is $\mathcal{D}_{+}=\mathcal{D}(T) \cap \mathcal{D}(S)$ and let $S_{\mid \mathcal{D}_{+}}$ and $T_{\mid \mathcal{D}_{+}}$be respectively the restrictions of $S$ and $T$ to $\mathcal{D}_{+}$. Then we have, from the above proposition, for all $x \in \mathcal{D}_{+}$:

$$
\begin{aligned}
(S+T) x=S_{\mid \mathcal{D}_{+}} x+T_{\mid \mathcal{D}_{+}} x & =A_{\mid \mathcal{D}_{+}} x+S(0)+B_{\mid \mathcal{D}_{+}} x+T(0) \\
& =\left(A_{\mid \mathcal{D}_{+}}+B_{\mid \mathcal{D}_{+}}\right) x+(S+T)(0) .
\end{aligned}
$$

This implies that:

$$
S+T=\left[A_{\mid \mathcal{D}_{+}}+B_{\mid \mathcal{D}_{+}}\right]+[(S+T)-(S+T)]
$$

Thus, $A_{\mid \mathcal{D}_{+}}+B_{\mid \mathcal{D}_{+}}$is a semiclosed selection of $S+T$. Hence, $S+T$ is semiclosed linear relation.

Let us denote by $\mathcal{D}_{\times}$the domain of $S T$. Then, $\mathcal{D}_{\times}=T^{-1}(\mathcal{D}(S))$ and for all $x \in \mathcal{D}_{\times}$we have:

$$
S T x=S(T x)=A B x+S T(0) .
$$

Hence, $A B$ is a semiclosed selection of $S T$ because both of $A$ and $B$ are semiclosed operators. On the other hand, we have $S T(0)=S(T(0))$ is a semiclosed subset of $H$. Therefore, $S T \in S C(H)$. 
This theorem provides the affirmative answer to the question formulated in [2] about the semiclosedness of product of two semiclosed linear relations and generalizes largely the Propositions 1.10 and 1.11 of [2].

\section{Corollary 3.3.}

1. If $S, T$ are closed relations, then $T+S$ and $T S$ are semiclosed linear relations.

2. If $T$ is a semiclosed relation such that $\mathcal{R}(T) \subset \mathcal{D}(T)$ and $n \in \mathbb{N}^{*}$, then $T^{n}$ is also semiclosed relation.

3. The set of semiclosed linear relations is the smallest class closed under sum and product.

\subsection{Kato Rellich Theorem for semiclosed linear relations}

In this paragraph, we give a new result about semiclosed linear relations which is a consequence of the Kato-Rellich theorem about relatively bounded (respectively relatively compact) linear operators. Before stating the theorem we shall make some definitions.

Definition 3.2. [3] Let $S, T \in L R(H)$. Then, $S$ is said to be $T$-bounded if $\mathcal{D}(T) \subset \mathcal{D}(S)$ and there exists a constant $c \geq 0$ such that

$$
\|S(x)\| \leq c(\|x\|+\|T(x)\|) \text { for all } x \in \mathcal{D}(T) .
$$

If $S$ is $T$-bounded, then the inf of all numbers $b \geq 0$ for which a constant $a \geq 0$ exists such that

$$
\|S(x)\| \leq a\|x\|+b\|T(x)\|, x \in \mathcal{D}(T),
$$

is called the $T$-bound of $S$.

Theorem 3.6. Let $S, T \in L R(H)$ such that $S(0) \subset T(0)$. If $T(0)$ is closed and $S$ is $T$-bounded with $T$-bound less than 1 , then

$$
S+T \in S C(H) \Leftrightarrow T \in S C(H) .
$$

Proof. We just have to note that $S(0) \subset T(0)$ implies that $(S+T)(0)=T(0)$ and then the theorem follows immediately from the Theorem 7 of [12] and the Theorem 3.6 of [13].

\subsection{Limit and infinite sum of semiclosed linear relations}

Let $T_{\varepsilon}$ and $S_{n}$ be two indexed collections of semiclosed linear relations on a Hilbert space $H$, with $\varepsilon>0$ and $n \in \mathbb{N}$. Suppose that $T_{\varepsilon}$ and $S_{n}$ have the same multivalued part $\mathcal{T}(0)$ which is assumed to be closed and independent of $\varepsilon$ and $n$ and let $H_{\varepsilon}$ and $G_{n}$ be respectively the auxiliary Hilbert spaces of $T_{\varepsilon}$ and $S_{n}$. Assume that there 
exists two Hilbert spaces $K_{1}$ and $K_{2}$ continuously embedded in $E_{\varepsilon}$ and $E_{n}$ for all $\varepsilon>0, n \in \mathbb{N}$, respectively such that for all $x \in K_{1}, \sup _{\varepsilon>0}\left\|T_{\varepsilon} x\right\|<+\infty$ and for all $x \in K_{2}, \sup _{N}\left\|\sum_{n=0}^{N} S_{n} x\right\|<+\infty$ for every $N \in \mathbb{N}$. Then the following result holds.

Theorem 3.7. If all of the above assumptions are satisfied, then:

1. the linear relation $T$ defined by $T x=\lim _{\varepsilon \rightarrow 0} T_{\varepsilon} x$ with the domain $\mathcal{D}(T)=\left\{x \in\left(\bigcap_{\varepsilon>0} \mathcal{D}\left(T_{\varepsilon}\right)\right) \cap K_{1}: \lim _{\varepsilon \rightarrow 0} T_{\varepsilon} x\right.$ exists in $\left.H\right\}$ is semiclosed on $H$,

2. the linear relation $S$ defined by $S x=\sum_{n=0}^{+\infty} S_{n} x$ with the domain

$$
\mathcal{D}(S)=\left\{x \in\left(\bigcap_{n \in \mathbb{N}} \mathcal{D}\left(S_{n}\right)\right) \cap K_{2}: \sum_{n=0}^{\infty} S_{n} x \text { exists in } H\right\} \text { is semiclosed on } H .
$$

Proof. 1. First note that $T(0)=\mathcal{T}(0)$ is closed and let us define on $\mathcal{D}(T)$ the following inner product:

$$
\begin{aligned}
(x, y) & =\langle x, y\rangle_{K_{1}}+\lim _{\varepsilon \rightarrow 0}\left\langle T_{\varepsilon} x, T_{\varepsilon} y\right\rangle_{H} \\
& =\langle x, y\rangle_{K_{1}}+\langle T x, T y\rangle_{H}
\end{aligned}
$$

and let $H_{T}=(\mathcal{D}(T),(.,)$.$) . Since K_{1}, H$ and $H_{\varepsilon}$ are Hilbert spaces and $T_{\varepsilon}$ is semiclosed for all $\varepsilon>0$, then $H_{T}$ is complete. In fact, let $\left(x_{n}\right)_{n}$ be a Cauchy sequence in $H_{T}$, then $\left(x_{n}\right)_{n}$ converges to $x$ in $K_{1}, H$ and $H_{\varepsilon}$, hence $x \in\left(\bigcap_{\varepsilon>0} \mathcal{D}\left(T_{\varepsilon}\right)\right) \cap K_{1}$ and from the semiclosedness of $T_{\varepsilon}$ we obtain: $T_{\varepsilon} x_{n}$ converges to $T_{\varepsilon} x$ for all $\varepsilon>0$. Since $\left(x_{n}\right)_{n}$ is a Cauchy sequence, there exists $\lambda>0$ such that:

$$
\left\|x_{n}\right\|_{H_{T}}=\left(x_{n}, x_{n}\right)^{1 / 2}<\lambda
$$

and

$$
\|x\|_{H_{T}}^{2}=\lim _{n \rightarrow+\infty}\left\|x_{n}\right\|_{K_{1}}^{2}+\lim _{\varepsilon \rightarrow 0} \lim _{n \rightarrow+\infty}\left\|T_{\varepsilon} x_{n}\right\|_{H}^{2}<2 \lambda^{2} .
$$

Hence, $x \in H_{T}$.

Let $\alpha>0$. Then, by the assumption $\sup _{\varepsilon>0}\left\|T_{\varepsilon} x\right\|_{H}<+\infty$ on $K_{1}$ and the uniform boundedness principle, there exists $j \in \mathbb{N}$ such that for all $n, m \geq j$ and $\varepsilon>0$,

$$
\left\|x_{n}-x_{m}\right\|_{H_{T}} \leq \frac{\alpha}{2} \text { and }\left\|T_{\varepsilon} x_{n}-T_{\varepsilon} x_{m}\right\|_{H_{T}} \leq \lambda \frac{\alpha}{2}
$$


Moreover, we have

$$
\begin{aligned}
\left\|x_{n}-x\right\|_{H_{T}} & =\left[\lim _{m \rightarrow+\infty}\left\|x_{n}-x_{m}\right\|_{K_{1}}^{2}+\lim _{\varepsilon \rightarrow 0} \lim _{m \rightarrow+\infty}\left\|T_{\varepsilon} x_{n}-T \varepsilon x_{m}\right\|_{H}^{2}\right]^{1 / 2} \\
& \leq \frac{\alpha}{2}\left(1+\lambda^{2}\right)^{1 / 2} .
\end{aligned}
$$

Consequently, $H_{T}$ is a Hilbert space, continuously embedded in $H$ and $T$ is continuous from $H_{T}$ onto $H$. Thus, we have from Corollary 3.2, $T$ is semiclosed.

2. Let $S x=\sum_{n=0}^{+\infty} S_{n} x$ with domain

$\mathcal{D}(S)=\left\{x \in\left(\bigcap_{n \in \mathbb{N}} \mathcal{D}\left(S_{n}\right)\right) \cap K_{2}: \sum_{n=0}^{\infty} S_{n} x\right.$ exists in $\left.H\right\}$.

Define $S_{N}=\sum_{n=0}^{N} S_{n}$ with domain $\mathcal{D}\left(S_{N}\right)=\left(\bigcap_{n=0}^{N} \mathcal{D}\left(S_{n}\right)\right) \cap K_{2}$. Then, $S_{N}$ is semiclosed linear relation with closed multivalued part and auxiliary Hilbert space $H_{S_{N}}=\left(\mathcal{D}\left(S_{N}\right),(., .)_{S_{N}}\right)$ where

$$
(x, y)_{S_{N}}=\langle x, y\rangle_{K_{2}}+\left\langle S_{N} x, S_{N} y\right\rangle \text { for all } x, y \in \mathcal{D}\left(S_{N}\right) .
$$

Obviously, $S x=\sum_{n=0}^{+\infty} S_{n} x=\lim _{N \rightarrow+\infty} S_{N} x$ and

$$
D(S)=\left\{x \in\left(\bigcap_{N \in \mathbb{N}} \mathcal{D}\left(S_{N}\right)\right) \cap K_{2}: \lim _{N \rightarrow+\infty} S_{N} x \text { exists in } H\right\} .
$$

Hence, we have from the first assertion and the fact that $S(0)=\mathcal{T}(0), S$ is semiclosed linear relation on $H$.

\section{Semiclosed linear relation with closed range}

There are many important applications of the closedness of the range in the spectral study of differential operators and also in the context of perturbation theory, we have investigated in this section semiclosed linear relations with closed range.

Theorem 4.1. Let $T \in S C(H)$. Then $\mathcal{R}(T)$ is closed if and only if $\mathcal{R}(T) \oplus N$ is closed for some semiclosed subspace $N$ in $H$.

Proof. If $\mathcal{R}(T)$ is closed in $H$, it is then sufficient to choose $N=\{0\}$ to have the stated result.

Conversely, suppose that there exists an semiclosed subspace $N$ of $H$ such that $\mathcal{R}(T) \oplus N$ is closed in $H$. Since $T \in S C(H)$, then by virtue of the Corollary 3.1, $\mathcal{R}(T)$ is always a semiclosed subspace of $H$. Therefore, by the assertion 3 of the Theorem $3.2 \mathcal{R}(T)$ is closed in $H$. 
In fact, semiclosed linear relations with closed null space and closed range in $H$ are closed linear relations on $H$.

Theorem 4.2. Let $T \in S C(H)$ such that $\mathcal{N}(T)$ and $\mathcal{R}(T)$ are closed in $H$, then $T$ is a closed linear relation.

Proof. Like $T \in S C(H)$, then the graph $G(T)$ of $T$ is semiclosed in $H \times H$. Moreover, we have:

$$
\begin{aligned}
& (H \times\{0\})+G(T)=H \times\{0\}+\{0\} \times \mathcal{R}(T), \\
& (H \times\{0\}) \cap G(T)=\mathcal{N}(T) \times\{0\}
\end{aligned}
$$

These two subspaces are closed in $H \times H$. Using the assertion 3 of Theorem 3.2 , we deduce that $G(T)$ is closed in $H \times H$ and consequently $T$ is a closed linear relation on $H$.

Theorem 4.3. Let $T \in S C(H)$ such that $\mathcal{R}(T)$ is closed in $H$. Then:

$$
\overline{G(T)}=G(T)+(\overline{\mathcal{N}(T)} \times\{0\}) .
$$

Proof. $G(T)$ is semiclosed, $H \times\{0\}$ and $H \times\{0\}+G(T)=H \times\{0\}+\{0\} \times R(T)$ are closed subspaces of $H \times H$. Let's put

$$
\begin{aligned}
H_{0} & =G(T)+\overline{G(T) \cap(H \times\{0\})}=G(T)+\overline{N(T) \times\{0\}} \\
& =G(T)+\overline{N(T)} \times\{0\} .
\end{aligned}
$$

$H_{0}$ is semiclosed in $H \times H$ and

$$
H_{0}+H \times\{0\} \subseteq H \times\{0\}+G(T)=H \times\{0\}+\{0\} \times R(T) \subseteq H_{0}+H \times\{0\} .
$$

Thus, $H_{0}+H \times\{0\}$ is closed and by virtue of Neubauer's lemma we find that $H_{0}$ is in fact a closed subspace of $H \times H$. On the other hand,

$$
G(T) \subseteq H_{0} \subseteq \overline{G(T)},
$$

so what $H_{0}=\overline{G(T)}$.

In the following, we will exploit the above result to give a new characterization of closable linear relations. Recall that a linear relation $T$ is said to be closable if and only if $T(0)$ is closed and $T_{s}$ is closable. Hence, if $T$ is supposed semiclosed on $H$ with $T(0)$ closed, then $T_{s}$ is also semiclosed in $H$, in addition, if we assume that $\mathcal{R}\left(T_{s}\right)$ is closed we obtain from the above theorem:

$$
\overline{G\left(T_{s}\right)}=G\left(T_{s}\right)+\left(\overline{\mathcal{N}\left(T_{s}\right)} \times\{0\}\right) .
$$


Theorem 4.4. Let $T \in S C(H)$ such that $T(0)$ and $\mathcal{R}\left(T_{s}\right)$ are closed in $H$. Then, $T$ is closable if and only if $\overline{\mathcal{N}(T)} \cap \mathcal{D}(T)=\mathcal{N}(T)$.

Proof. Firstly, note that if $T(0)$ is closed, then $\mathcal{N}(T)=\mathcal{N}\left(T_{s}\right)$. Let $T$ be closable (i.e $T_{s}$ is closable) and $x \in \overline{\mathcal{N}(T)} \cap \mathcal{D}(T)=\overline{\mathcal{N}\left(T_{s}\right)} \cap \mathcal{D}\left(T_{s}\right)$, then there exists a sequence $\left(x_{n}\right)_{n \in \mathbb{N}}$ in $\mathcal{N}\left(T_{s}\right)$ that converges to $x$ in $H$. So, $\left(x-x_{n}\right) \rightarrow 0$ and $T_{s}\left(x-x_{n}\right)=T_{s} x \rightarrow T_{s} x$, from where $T_{s} x=0$ and $x \in \mathcal{N}\left(T_{s}\right)=\mathcal{N}(T)$.

Conversely, let $(0, y) \in \overline{G\left(T_{s}\right)}=G\left(T_{s}\right)+\left(\overline{\mathcal{N}\left(T_{s}\right)} \times\{0\}\right)$. Then there is $x \in$ $\mathcal{D}\left(T_{s}\right)$ and $t \in \overline{\mathcal{N}\left(T_{s}\right)}$ such that $x+t=0$ and $T_{s} x=y$. Therefore, $x=-t \in$ $\overline{\mathcal{N}\left(T_{s}\right)} \cap \mathcal{D}\left(T_{s}\right)=\mathcal{N}\left(T_{s}\right)$ and $y=T_{s} x=0$. Which means that $\overline{G\left(T_{s}\right)}$ is the graph of a linear operator, ie $T_{s}$ is closable on $H$. Hence $T$ is closable linear relation

\section{REF E R E N C ES}

1. T. Alvarez, R.W. Cross, D. Wilcox: Multivalued Fredholm type operators with abstract generalised inverses, J. Math. Anal. Appl. 261 (2001), 403-417.

2. T. Alvarez, D. Wilcox: The Baire property and the domain of iterates of a paracomplete linear relation, J. Operator theory. 66 (2011), no. 2, 451-464.

3. R.W. CRoss: Multivalued Linear Operators, Marcel Dekker, New York 1998.

4. M.R. EmBRY: Factorization of operators on Banach spaces, Proc. Amer. Math. Soc. 38 (1973), 587-590.

5. V.P. Fonf, V.V. SheVchiK: Operators on dense embedding and quasicomplements of subspaces of Banach spaces, Arch. Math. 62 (1994), 539-544.

6. A. Gherbi, B. Messirdi, M. Benharrat: Quotient operators: new generation of linear operators, Funct. Anal. Approx. Comput. 7 (2015), no. 1, 85-93.

7. A. Gherbi, B. Messirdi: Quotient of linear relations and applications, AsianEur. J. Math. 12 (2019), no. 1, 01-20.

8. W. E. Kaufman: Semiclosed operators in Hilbert space, Proc. Amer. Math. Soc. 76 (1979), 67-73.

9. W. E. Kaufman: Closed operators and pure contractions in Hilbert space, Proc. Amer. Math. Soc. 87 (1983), 83-87.

10. J.-P. LABRoussE: Les operateurs quasi-Fredholm: une generalisation des operateurs semi-Fredholm, Rend. Circ. Mat. Palermo 29 (1980), 161-258.

11. J.-Ph. Labrousse, A. Sandovici, H. De Snoo: The Kato decompsition of quasi-Fredholm relations, Operators and Matrices. 4 (2010), no. 1, 1-51.

12. S. Messirdi, M. DjaA, B. Messirdi: Stability of almost closed operators on a Hilbert space, Sarajevo J. Math. 5 (2009), no. 17, 133-141.

13. S. Yuming, X. Guixin, R. Guojing: Boundedness and closedness of linear relations, Linear Multilinear Algebra. 66 (2018), no. 2, 309-333. 